\title{
Topological responses from chiral anomaly in multi-Weyl semimetals
}

\author{
Ze-Min Huang, Jianhui Zhou, and Shun-Qing Shen \\ Department of Physics, The University of Hong Kong, Pokfulam Road, Hong Kong, China
}

(Received 5 May 2017; published 4 August 2017)

\begin{abstract}
Multi-Weyl semimetals are a kind of topological phase of matter with discrete Weyl nodes characterized by multiple monopole charges, in which the chiral anomaly, the anomalous nonconservation of an axial current, occurs in the presence of electric and magnetic fields. Electronic transport properties related to the chiral anomaly in the presence of both electromagnetic fields and axial electromagnetic fields in multi-Weyl semimetals are systematically studied. It has been found that the anomalous Hall conductivity has a modification linear in the axial vector potential from inhomogeneous strains. The axial electric field leads to an axial Hall current that is proportional to the distance of Weyl nodes in momentum space. This axial current may generate chirality accumulation of Weyl fermions through delicately engineering the axial electromagnetic fields even in the absence of external electromagnetic fields. Therefore this work provides a nonmagnetic mechanism of generation of chirality accumulation in Weyl semimetals and might shed new light on the application of Weyl semimetals in the emerging field of valleytronics.
\end{abstract}

DOI: 10.1103/PhysRevB.96.085201

\section{INTRODUCTION}

Weyl semimetals are materials with a finite number of band touching points, Weyl nodes, in the three-dimensional Brillouin zone [1-4]. These Weyl nodes can be viewed as magnetic monopoles in momentum space [5,6], which can lead to various anomalous transport properties, including static and dynamical chiral magnetic effects [7-20], anomalous Hall effect (AHE) [21,22], chirality-dependent Hall effect [23,24], nonlocal transport signature [25], and anomalous magnetoresistance [26-35]. However, most of these studies focused on single-Weyl semimetals, whose energy dispersions are linear in wave vectors and monopole charge equals \pm 1 . Recently, there are proposals of multi-Weyl fermions [36-38], with multiple monopole charges and nonlinear dispersion relation. For example, first-principles calculations suggest that a pair of double-Weyl nodes exist in $\mathrm{HgCr}_{2} \mathrm{Se}_{4}$ [36] and $\mathrm{SrSi}_{2}$ [38]. It has been shown that the nonlinear dispersion and double monopole charges of double-Weyl semimetals have led to unconventional correlation effects [39-42], magnetotransport [15,43-45], and thermoelectric transport [46]. In this work, our prime aim is to systematically investigate topological transport properties of multi-Weyl fermions associated with the chiral anomaly.

The chiral anomaly in single-Weyl semimetals had been derived in several different ways [26,47-49], but derivation of the chiral anomaly in multi-Weyl semimetals is still absent. In the intuitive and physical derivation given by Nielsen and Ninomiya [26], helicity plays an important role. Namely, for massless fermions in a homogeneous magnetic field $\mathbf{B}$, the spins are preferentially aligned along $\mathbf{B}$. Then the left-handed and the right-handed fermions are accelerated under an electric field $\mathbf{E}$, resulting in Adler-Bell-Jackiw anomaly or chiral anomaly [47]. When it comes to multi-Weyl semimetals, the Lorentz invariance is broken such that helicity is not a well-defined quantity. Hence the specific forms of the chiral anomaly and Jacobian under a chiral transformation are still absent, which call for a detailed derivation. Since the chiral anomaly closely relates to topological responses $[1,10]$, the chiral anomaly shall assist us to construct the corresponding effective action and then to investigate the relevant topological responses.

In addition, axial magnetic fields, as large as $300 \mathrm{~T}$, can be simulated by elastic deformations of lattice and couple to Dirac fermions in graphene [50-52]. For single-Weyl semimetals, three-dimensional counterparts of graphene, it has been demonstrated that axial magnetic fields emerge from strain fields, nonuniform magnetizations, or topological defects [53-55]. Theoretical investigations show that the axial gauge fields can induce anomalous topological properties in single-Weyl semimetals, including chiral pseudomagnetic effect [11,56-58], plasmon-magnon coupling [53], phonon Hall viscosity [54,59], emergent gravity [60,61], and chiral magnetic plasmons [62]. Therefore one would naturally wonder whether the interplay between electromagnetic fields and axial gauge fields occurs in multi-Weyl semimetals.

In this paper, we apply the quantum-field-theory approach to explore the topological responses of multi-Weyl semimetals to the electromagnetic fields as well as the axial electromagnetic fields. We find that the strain fields make a significant contribution linear in the axial vector potential $\mathbf{A}^{5}$ to the anomalous Hall conductivity. It has been shown that both an axial magnetic field and an axial electric field lead to axial currents proportional to the chiral chemical potential $b_{0}$ and the distance of Weyl nodes in momentum space $\mathbf{b}$, respectively. Consequently, the chirality accumulation of Weyl fermions can be achieved at the surfaces of a sample completely through engineering the axial fields. In addition, the realization and detection of the strain-induced AHE, the axial currents and the resulting chirality accumulation in double-Weyl semimetals are discussed.

The rest of this paper is organized as follows. In Sec. II, we introduce the effective Hamiltonian and the Lagrangian density for multi-Weyl semimetals and discuss the topological invariant. In Sec. III, we derive the chiral anomaly equations for multi-Weyl semimetals in the presence of both the electromagnetic fields and the axial gauge fields. In Sec. IV, the topological responses due to the chiral anomaly are present. The realizations and detections of the strain-induced 
AHE, electric/axial currents and chirality accumulation in double-Weyl semimetals are proposed. In Sec. V, the main results of this paper are summarized. Finally, in Appendixes, we give a detailed calculations of the effective action and the chiral anomaly equations.

\section{MODEL FOR MULTI-WEYL SEMIMETALS}

We start with the following Hamiltonian for multi-Weyl semimetals containing a pair of Weyl nodes with multiple monopole charges [37,44,63],

$$
H=\left(\begin{array}{cc}
H_{+} & 0 \\
0 & H_{-}
\end{array}\right),
$$

with

$$
H_{s}=s v\left[(\mathbf{p}+s \mathbf{b})_{3} \sigma^{3}+w^{-1}(\mathbf{p}+s \mathbf{b})_{+}^{n} \sigma^{-}+w^{-1}(\mathbf{p}+s \mathbf{b})_{-}^{n} \sigma^{+}\right],
$$

where $v$ is the effective velocity, $w$ is a material-dependent parameter, $\mathbf{p}$ denotes for momentum, $p_{ \pm}=\left(p_{1} \pm i p_{2}\right) / \sqrt{2}$, and $\mathbf{b}$ characterizes the distance between Weyl nodes with opposite chirality in momentum space. $s= \pm 1$ are the chiralities of Weyl nodes. The Weyl node $s$ locates at $-s \mathbf{b}$ in momentum space. $\sigma^{i}$ are the Pauli matrices $(i=1,2,3), \sigma^{ \pm}=\left(\sigma^{1} \pm\right.$ $\left.i \sigma^{2}\right) / \sqrt{2}$ and $n$ is a positive integer. It has been pointed out that both double- $(n=2)$ and triple- $(n=3)$ Weyl semimetals are protected by $C_{4}$ and $C_{6}$ symmetry, respectively. But other more higher order band-crossing points are not protected by $n$-fold rotational symmetry [37]. Thus the possible values of $n$ for multi-Weyl semimetals are $n=2,3$, which might be detected by quantum transport measurement [43]. Their corresponding density of states (DOS) is present in Table I. One finds that, compared with single-Weyl semimetals, the DOS of either double- or triple-Weyl semimetals possesses greatly different dependence of energy. To be specific, the DOS obeys the law $|E|^{2 / n}$ up to a material-dependent constant. In addition, the DOS for Weyl semimetals with $n=1,2,3$ vanishes identically at Weyl nodes.

For each Weyl node, the winding number can be defined by [6]

$$
N_{s}=-\frac{1}{3 !(2 \pi i)^{2}} \int_{S^{3}} \operatorname{tr}\left(G^{\tau} d G^{\tau-1}\right)^{3},
$$

where $G^{\tau}$ is the imaginary-time Green's function and $S^{3}$ means integrating over a three-dimensional sphere in frequency-momentum space enclose this Weyl node. tr acts on the degree of freedom $\sigma^{i}$. For our model in Eq. (1), one finds

$$
N_{s}=s n \text {. }
$$

TABLE I. Monopole charge $n$ and density of states at zero temperature for Weyl semimetals with $n=1,2,3 . \Gamma(x)$ is the gamma function.

\begin{tabular}{cc}
\hline \hline$n$ & DOS \\
\hline 1 & $\frac{w^{2}}{2 \pi^{2} v^{3}} E^{2}$ \\
2 & $\frac{\sqrt{2} w}{8 \pi v^{2}}|E|$ \\
3 & {$\left[\frac{w^{2 / 3} \Gamma(1 / 3)^{3}}{2^{8 / 3} \sqrt{3} \pi^{3} v^{5 / 3}}\right]|E|^{2 / 3}$} \\
\hline \hline
\end{tabular}

Although the winding number $N_{s}$ defined here is equivalent to the Chern number [64], to make a closer connection to the effective action below, we shall adopt the winding number rather than the Chern number throughout this paper. The sum of $N_{s}\left(N=\Sigma_{s} N_{s}\right)$ can also be obtained from integrating over the whole area $p_{0,1,2} \in(-\infty, \infty)$ with fixed $p_{3}$,

$$
N\left(p_{3}\right)=n\left[\theta\left(p_{3}+b_{3}\right)-\theta\left(p_{3}-b_{3}\right)\right],
$$

where $\theta(x)$ is the Heaviside step function: $\theta(x)=1$ for $x \geqslant 0$ and otherwise vanishes. Equation (4) shall manifest itself in the effective action. From Eq. (4), one can determine the locations of Weyl nodes: $\frac{d N}{d p_{3}}= \pm n \delta\left(p_{3} \pm b_{3}\right)$.

As mentioned above, due to the explicit violation of Lorentz symmetry, the helicity is not well-defined and the chiral condition is invalid as well: $\gamma^{5} \Psi_{ \pm}= \pm \Psi_{ \pm}$. For single-Weyl nodes, the chirality is equivalent to the sign of the winding number. Analogically, we adopt the latter as a generalized definition for the chirality. With this respect, the $\gamma^{5}$ matrix can be defined by setting $s$ as the eigenvalue and $\Psi_{ \pm}$ as the eigenfunctions. For later convenience, we write the corresponding Lagrangian density as

$$
\begin{aligned}
\mathcal{L}= & \bar{\Psi}\left\{\gamma^{0}\left(p_{0}-e A_{0}-e A_{0}^{5} \gamma^{5}+m_{0}+b_{0} \gamma^{5}\right)\right. \\
& +\gamma^{3}\left(p_{3}-e A_{3}-e A_{3}^{5} \gamma^{5}+m_{3}+b_{3} \gamma^{5}\right) \\
& +\frac{1}{w}\left[\gamma^{+}\left(p-e A-e A^{5} \gamma^{5}+m+b \gamma^{5}\right)_{+}^{n}\right. \\
& \left.\left.+\gamma^{-}\left(p-e A-e A^{5} \gamma^{5}+m+b \gamma^{5}\right)_{-}^{n}\right]\right\} \Psi,
\end{aligned}
$$

where $\mu, v=0,1,2,3, v$ has been absorbed into $p_{\mu}=$ $\left(p_{0},-\mathbf{p}\right), b_{0}$ is the chiral chemical potential in a steady state not the energy difference between two Weyl nodes $[8,65], m_{0}$ is the averaged chemical potential and $-\mathbf{m}$ is the center of momentum of Weyl nodes. $\mu_{s}=m_{0}+s b_{0}$ is the chiralitydependent chemical potential. $\gamma^{\mu}$ is the gamma matrix, satisfying $\left\{\gamma^{\mu}, \gamma^{\nu}\right\}=2 g^{\mu \nu}$ with $g^{\mu \nu}=\operatorname{diag}\{1,-1,-1,-1\}, \gamma^{i}=$ $\sigma^{i} \otimes\left(-i \tau^{2}\right), \gamma^{0}=\sigma^{0} \otimes \tau^{1}, \gamma^{ \pm}=\left(\gamma^{1} \mp i \gamma^{2}\right) / \sqrt{2}$, and $\gamma^{5}=$ $\sigma^{0} \otimes \tau^{3} . \Psi$ is a Grassmann number, $\Psi=\left(\Psi_{+}, \Psi_{-}\right)^{T}$ and $\bar{\Psi}=\Psi^{\dagger} \gamma^{0} . F_{\mu \nu}=\partial_{\mu} A_{\nu}-\partial_{\nu} A_{\mu}$ is the field strength tensor for electromagnetic fields. $A_{\mu}^{5}$ is the axial vector potential. The field strength tensor for $A_{\mu}^{5}$ is defined as $F_{\mu \nu}^{5}=\partial_{\mu} A_{\nu}^{5}-\partial_{\nu} A_{\mu}^{5}$. In analogy to Maxwell's equations for electromagnetic fields, it is instructive to examine the dynamics of axial electromagnetic fields. The Bianchi identity for $F_{\mu \nu}^{5}$ gives rise to $\nabla \cdot \mathbf{B}_{5}=0$ and $\partial_{t} \mathbf{B}_{5}=-\nabla \times \mathbf{E}_{5}$, which are identical to the ones for $F_{\mu \nu}$. However, the sourceless free equations of motion when $A_{0}^{5}=0$ become

$$
\nabla \cdot \mathbf{E}_{5}=\partial_{t}\left(\nabla \cdot \mathbf{A}^{5}\right)
$$

and

$$
\partial_{t} \mathbf{E}_{5}-\nabla \times \mathbf{B}_{5}=\nabla\left(\nabla \cdot \mathbf{A}^{5}\right)-\nabla^{2} \mathbf{A}^{5}+\partial_{t}^{2} \mathbf{A}^{5} .
$$

Since $A_{\mu}^{5}$ is observable and single valued $[54,58,66]$, one thus has no redundant gauge freedom to ensure $\nabla \cdot \mathbf{A}^{5}=0$. It should been pointed out that in general, the strain fields should not only produce an axial gauge potential but also modify the local geometry metric [60,61]. In this work, we mainly focus on the impact of axial gauge potentials on Weyl fermions. Note that the 4 -vector $b_{\mu}=\left(b_{0},-\mathbf{b}\right)$ can 
be eliminated by performing a large chiral transformation, $\Psi \rightarrow e^{i\left(b_{\mu} x^{\mu}\right) \gamma^{5}} \Psi$. Due to Fujikawa's uncertainty principle $[47,48]$, such a chiral transformation would give rise to a term from the path-integral measure. However, the specific form of this Jacobian is still absent for multi-Weyl semimetals. Note that we have neglected the conventional action for classical electrodynamics $-F_{\mu \nu} F^{\mu \nu} / 4$ in Eq. (5).

\section{CHIRAL ANOMALY IN MULTI-WEYL SEMIMETALS}

In this section, the equations for the chiral anomaly in multi-Weyl semimetals in the presence of both electromagnetic fields and axial fields are derived by using the Fujikawa's method [48]. For a heuristic purpose, we outline the key points in the application of the Fujikawa's method in derivation of the chiral anomaly for relativistic fermions. Since the Jacobian of the chiral-transformation is divergent like $\ln J \propto \operatorname{Tr}\left[\gamma^{5} \delta(x-x)\right.$ ] [69], one needs to introduce a regulator, i.e., $\lim _{M \rightarrow \infty} \exp \left(-|\not D|^{2} / M^{2}\right)$ where $\not D=\gamma^{\mu} D_{\mu}$ is the Dirac operator and $M$ is a large positive parameter for regularization. After long and straightforward calculations, one gets $\ln J=-i e^{2} \epsilon^{\mu \nu \alpha \beta} F_{\mu \nu} F_{\alpha \beta} / 16 \pi^{2}$, where $\epsilon^{\mu \nu \rho \sigma}$ is the four-rank antisymmetric Levi-Civita symbol.

Similarly, for multi-Weyl semimetals under electromagnetic fields, the regulated Jacobian reads

$$
J[\beta]=\exp \left\{-2 i \beta \int \lim _{M \rightarrow \infty} \operatorname{tr}\left[\gamma^{5} e^{-\frac{\left|i D^{n}\right|^{2}}{M^{2}}} \delta(x-x)\right]\right\},
$$

where $\beta$ comes from chiral transformation of $\Psi \rightarrow e^{i \beta \gamma^{5}} \Psi$, and the operator $i \not D^{n}$ is defined as

$$
i D_{0,3}^{n}(A)=p_{0,3}-e A_{0,3}
$$

and

$$
i D_{ \pm}^{n}(A)=\left(p_{ \pm}-e A_{ \pm}\right)^{n} / w
$$

with $A_{ \pm}=\left(A_{1} \pm i A_{2}\right) / \sqrt{2}$ being the components of the vector potential. By performing a Fourier's transformation and a Wick's rotation to the Euclidean spacetime, the Jacobian can be recast as

$$
\begin{aligned}
\frac{\ln J[\beta]}{-2 i \beta}= & \epsilon_{E}^{\mu \nu \rho \sigma} \lim _{M \rightarrow \infty} \int \frac{d^{d} k}{(2 \pi)^{d}} e^{-\frac{k_{0}^{2}+k_{3}^{2}+\left(k_{1}^{2}+k_{2}^{2}\right)^{n} / 2^{n-1} w^{2}}{M^{2}}} \\
& \times \frac{\left[i D_{\mu}^{n}, i D_{\nu}^{n}\right]\left[i D_{\rho}^{n}, i D_{\sigma}^{n}\right]}{4 M^{4}}
\end{aligned}
$$

where we have used the identity $4 \epsilon_{E}^{\mu \nu \rho \sigma}=\operatorname{tr}\left(\gamma^{5} \gamma_{E}^{\mu} \gamma_{E}^{\nu} \gamma_{E}^{\rho} \gamma_{E}^{\sigma}\right)$, the subscript $E$ denotes for the Euclidean spacetime. Rescaling momentum variables as $k_{0,3} \rightarrow M k_{0,3}$ and $k_{1,2} \rightarrow$ $(w M)^{1 / n} k_{1,2}$, one finds

$$
\begin{aligned}
\frac{\ln J}{-2 i \beta}= & \frac{\epsilon_{E}^{\mu \nu \rho \sigma}}{2} \lim _{M \rightarrow \infty} \int \frac{d^{d} k}{(2 \pi)^{d}} e^{-\left[k_{0}^{2}+k_{3}^{2}+\left(k_{1}^{2}+k_{2}^{2}\right)^{n} / 2^{n-1}\right]} \\
& \times\left[\sum_{m=1}^{n-1} T_{m}(w M)^{-2 m / n} F_{\mu \nu}\left(\partial_{+} \partial_{-}\right)^{m} F_{\rho \sigma}\right. \\
& \left.\times\left|D_{+} D_{-}\right|^{n-m-1}-n^{2} F_{\mu \nu} F_{\rho \sigma}\left|D_{+} D_{-}\right|^{n-1}\right],
\end{aligned}
$$

where $T_{m}$ is a combination coefficient, for example, $T_{m}=$ $\delta_{m 1}$ for double-Weyl semimetals. Several remarks about the manifestations of Lorentz symmetry breaking in this derivation are in order here. First, the decay factor of $\left\{k_{0}^{2}+k_{3}^{2}+2 w^{-2}\left[\left(k_{1}^{2}+k_{2}^{2}\right) / 2\right]^{n}\right\} / M^{2}$ instead of $k_{\mu} k^{\mu} / M^{2}$ in single-Weyl semimetals requires $k_{\mu}$ to be scaled anisotropically. Second, commutators of covariant derivative are not covariant under Lorentz transformation. For example, $\quad w\left[i D_{0}^{n=2}, i D_{ \pm}^{n=2}\right]=2 F_{0 \pm} D_{ \pm}+\left(\partial_{ \pm} F_{0 \pm}\right)$ for doubleWeyl semimetals rather than $\left[D_{\mu}, D_{\nu}\right]=i e F_{\mu \nu}$ for singleWeyl semimetals. As a result, there exists a factor of $\left|D_{+} D_{-}\right|^{n-1}$ in the second term in the third line, which disappears in single-Weyl semimetals. In addition, the group of terms in the second line in Eq. (12) completely originates from the breaking of Lorentz symmetry and thus is forbidden in single-Weyl semimetals.

After taking the limit $M \rightarrow \infty$, all the terms in the second line in Eq. (12) are suppressed. Thus one only needs to pick up the leading term of order $M^{0}$ and finally gets the Jacobian for multi-Weyl semimetals as (the derivations are given in Appendixes A and B)

$$
\ln J[\beta]=-i \frac{n e^{2}}{16 \pi^{2}} \int \beta \epsilon^{\mu \nu \alpha \beta} F_{\mu \nu} F_{\alpha \beta},
$$

where coefficient $n$ refers to the winding number defined in Eq. (2). Interestingly, Eq. (13) differs from the counterpart of single-Weyl semimetals by a factor of $n$. It is consistent with the intuitive picture of the chiral anomaly in the language of the chiral zeroth Landau levels in the presence of a magnetic field along the $z$ axis. In fact, there are $n$ chiral Landau levels crossing the zero energy for multi-Weyl semimetals in Eq. (1) [44].

Let us turn to evaluate the Jacobian in the presence of both electromagnetic fields and axial fields. In order to derive the corresponding Jacobian from the one only in the presence of electromagnetic fields in Eq. (13), we consider the following model:

$$
\mathcal{L}_{\mathrm{I}}=\sum_{a=1,2} \bar{\Psi}_{a} \gamma^{\mu} i D_{\mu}^{n}\left[A+(-1)^{a} A^{5}\right] \Psi_{a},
$$

where $\Psi_{a}$ denotes for the four-component Dirac spinor $\left(\Psi_{a+}, \Psi_{a-}\right)^{T}$, subscript $a=1,2$ labels these two Dirac spinors, and \pm stands for valley or Weyl node degree of freedom. Obviously, there are no axial gauge fields that couple to $\Psi_{1}$ and $\Psi_{2}$, which implies the U(1) symmetry. In addition, the effective electromagnetic fields couple to Dirac spinors $\Psi_{1}$ and $\Psi_{2}$ differently through $A_{\mu}-A_{\mu}^{5}$ and $A_{\mu}+A_{\mu}^{5}$, respectively. Thus one could obtain the Jacobians for $\Psi_{1}$ and $\Psi_{2}$ from the one in Eq. (13).

Alternatively, this Lagrangian density can also be written as follows:

$$
\mathcal{L}_{\mathrm{II}}=\sum_{a \neq b=1}^{2} \bar{\Psi}_{a b} \gamma^{\mu} i D_{\mu}^{n}\left(A-\epsilon^{a b} A^{5} \gamma^{5}\right) \Psi_{a b},
$$

where $\Psi_{a b}=\left(\Psi_{a+}, \Psi_{b-}\right)^{T}$ with $a, b=1,2$ and $a \neq b$. $D_{\mu}^{n}\left(A \pm A^{5} \gamma^{5}\right)$ is obtained from $D_{\mu}^{n}(A)$ by replacing $A_{\mu}$ with $A_{\mu} \pm A_{\mu}^{5} \gamma^{5} \cdot \epsilon^{12}=-\epsilon^{21}=1$. One can clearly recognize an axial gauge field $A^{5} \gamma^{5}$. Hence, the Jacobians for fields $\Psi_{a b}$ in $\mathcal{L}_{\text {II }}$ under $\mathrm{U}(1)$ and the chiral transformation are defined as 
$J_{\mathrm{U}}\left(A-\epsilon^{a b} A^{5} \gamma^{5}\right)$ and $J_{\mathrm{c}}\left(A-\epsilon^{a b} A_{5} \gamma^{5}\right)$, respectively. Since $\mathcal{L}_{\mathrm{I}}$ is equivalent to $\mathcal{L}_{\mathrm{II}}$, they should have the same the variations of action $\delta S_{\text {I }}=\delta S_{\text {II }}$ under some transformation. To be specific, under the transformations of $\Psi_{1} \rightarrow e^{i \beta} \Psi_{1}$ and $\Psi_{2} \rightarrow e^{-i \beta} \Psi_{2}$, the variations of action $S_{\mathrm{I}}$ and $S_{\mathrm{II}}$ are

$$
\delta S_{\mathrm{I}}^{(1)}=0
$$

and

$$
\delta S_{\mathrm{II}}^{(1)}=\ln J_{\mathrm{c}}\left(A-A^{5} \gamma^{5}\right)-\ln J_{\mathrm{c}}\left(A+A^{5} \gamma^{5}\right) .
$$

Note that $\delta S_{\mathrm{I}}^{(1)}=0$ is due to the $\mathrm{U}(1)$ symmetry in $\mathcal{L}_{\mathrm{I}}$. The implementation of $\Psi_{1,2} \rightarrow e^{i \beta \gamma^{5}} \Psi_{1,2}$ leads to

$$
\delta S_{\mathrm{I}}^{(2)}=-i \frac{n e^{2}}{8 \pi^{2}} \int \beta \epsilon^{\mu \nu \rho \sigma}\left(F_{\mu \nu} F_{\rho \sigma}+F_{\mu \nu}^{5} F_{\rho \sigma}^{5}\right)
$$

and

$$
\delta S_{\mathrm{II}}^{(2)}=\ln J_{\mathrm{c}}\left(A-A^{5} \gamma^{5}\right)+\ln J_{\mathrm{c}}\left(A+A^{5} \gamma^{5}\right) .
$$

Thus one gets the Jacobian

$$
\ln J_{\mathrm{c}}\left(A \pm A^{5} \gamma^{5}\right)=-\frac{i n e^{2} \epsilon^{\mu \nu \rho \sigma}}{16 \pi^{2}} \int \beta\left(F_{\mu \nu} F_{\rho \sigma}+F_{\mu \nu}^{5} F_{\rho \sigma}^{5}\right) \text {. }
$$

Similarly, we at first perform transformations $\Psi_{1,2} \rightarrow e^{i \beta} \Psi_{1,2}$ and obtain

$$
\delta S=\ln J_{\mathrm{U}}\left(A+A^{5} \gamma^{5}\right)+\ln J_{\mathrm{U}}\left(A-A^{5} \gamma^{5}\right)=0 .
$$

Then, we carry out another transformations: $\Psi_{1} \rightarrow e^{i \beta \gamma^{5}} \Psi_{1}$ and $\Psi_{2} \rightarrow e^{-i \beta \gamma^{5}} \Psi_{2}$, yielding

$$
\begin{aligned}
\delta S= & -i \frac{n e^{2}}{16 \pi^{2}} \int \beta \epsilon^{\mu \nu \alpha \beta}\left[\left(F_{\mu \nu}-F_{\mu \nu}^{5}\right)\left(F_{\alpha \beta}-F_{\alpha \beta}^{5}\right)\right. \\
& \left.-\left(F_{\mu \nu}+F_{\mu \nu}^{5}\right)\left(F_{\alpha \beta}+F_{\alpha \beta}^{5}\right)\right] \\
= & \ln J_{\mathrm{U}}\left(A-A^{5} \gamma^{5}\right)-\ln J_{\mathrm{U}}\left(A+A^{5} \gamma^{5}\right) .
\end{aligned}
$$

Combining Eqs. (21) and (22), one finds the Jacobian for U(1) transformation

$$
\ln J_{\mathrm{U}}\left(A \mp A^{5} \gamma^{5}\right)= \pm i \frac{n e^{2} \epsilon^{\mu \nu \rho \sigma}}{8 \pi^{2}} \int \beta F_{\mu \nu} F_{\rho \sigma}^{5} .
$$

With the help of $J_{\mathrm{U}}$ and $J_{\mathrm{c}}$, it is straightforward to derive the continuity equations for the electric current and the axial current for multi-Weyl semimetals

$$
\partial_{\mu} j^{\mu}=\frac{n e^{3}}{8 \pi^{2}} \epsilon^{\mu \nu \rho \sigma} F_{\mu \nu} F_{\rho \sigma}^{5}
$$

and

$$
\partial_{\mu} j^{5 \mu}=\frac{n e^{3}}{16 \pi^{2}} \epsilon^{\mu \nu \rho \sigma}\left(F_{\mu \nu} F_{\rho \sigma}+F_{\mu \nu}^{5} F_{\rho \sigma}^{5}\right),
$$

or in terms of $j_{ \pm}^{\mu}$ and $\mathbf{E}, \mathbf{E}_{5}, \mathbf{B}, \mathbf{B}_{5}$,

$$
\partial_{\mu} j_{s}^{\mu}=-s \frac{n e^{3}}{4 \pi^{2}} \mathbf{E}_{s} \cdot \mathbf{B}_{s}
$$

where $\mathbf{E}_{s} \equiv \mathbf{E}+s \mathbf{E}_{5}$ and $\mathbf{B}_{s} \equiv \mathbf{B}+s \mathbf{B}_{5}$ are the effective electric field and the effective magnetic field near the Weyl node $s . j_{s}^{\mu}=\left(j_{s}^{0}, \mathbf{j}_{s}\right)$ refers to the current near the Weyl node. Eq. (24) indicates the breaking of U(1) symmetry and the local charge nonconservation. Such an anomalous effect from $\mathbf{E}_{5} \cdot \mathbf{B}$ was attributed to the modulation of the band structure. To be specific, the band structure of the bulk is modified by strain fields through compressing or stretching an infinite crystal such that the chemical potential varies in order to accommodate these fixed number of electrons [58]. The local charge nonconservation due to $\mathbf{E} \cdot \mathbf{B}_{5}$ might originate from the charge transfer between bulk and boundary [58]. For $F_{\mu \nu}^{5}=0$, the right hand side of Eq. (24) vanishes, whereas Eq. (25) will reduce to the conventional chiral anomaly equation [47]. It is worth noting that this set of anomaly equations is known as the covariant anomaly in high-energy physics [48,70] and acts as the starting point to discuss topological responses of single-Weyl semimetals $[53,57,58,62]$. It is clear that the chiral anomaly equation in Eq. (26) differs from the counterparts for singleWeyl semimetals by a winding number $n$ and paves the way to study the physics induced by the chiral anomaly in multi-Weyl semimetals. It is one of the main results in this work.

Actually, these anomaly equations in Eq. (26) can be intuitively understood through the lowest chiral Landau levels [26]. For simplicity, we consider the case that the strengths of the electric/magnetic fields are stronger than those of the axial electric/magnetic fields. When an effective magnetic field $B_{s}$ is applied along $z$ direction, the energy dispersion of the $n$-fold degenerate lowest chiral Landau levels for Weyl node $s$ is $s k_{z}$. According to the semiclassical equation of motion of electrons, adiabatically turning on an additional electric field $E_{s}$ along $z$ axis leads to a change of the momentum of quasiparticles $\Delta p_{z}=-s e E_{s} \Delta t$ over a period of time $\Delta t$. Hence the total variation of charge density for all of the $n$ chiral Landau levels is given as

$$
\begin{aligned}
\frac{\Delta j_{s}^{0}}{\triangle t} & =(-e) n\left(\frac{\Delta p_{z}}{2 \pi \Delta t}\right)\left(-\frac{e B_{s}}{2 \pi}\right) \\
& =-s \frac{n e^{3}}{4 \pi^{2}} E_{s} B_{s},
\end{aligned}
$$

where the last set of parentheses in the first line denotes for the degeneracy of each chiral Landau level. This can be recast in a covariant form as: $\partial_{\mu} j_{s}^{\mu}=-s \frac{n e^{3}}{4 \pi^{2}} \mathbf{E}_{s} \cdot \mathbf{B}_{s}$, which is identical to Eq. (26). It has also been numerically demonstrated that even tilting the magnetic field away from $z$ axis, one still obtains $n$ chiral Landau levels crossing the zero energy [44]. Carrying out the similar procedure above, one finally reproduces Eq. (26) as well. It should be noted that the chiral anomaly equation in Eq. (26) can also be straightforwardly verified within the semiclassical chiral kinetic theory $[5,49]$.

\section{TOPOLOGICAL RESPONSES}

In this section, the topological responses of multi-Weyl fermions to the electromagnetic field and the axial electromagnetic field are derived. In the end, the realizations of the strain-induced AHE and the anomalous axial Hall effect are proposed in Weyl semimetals.

As a minimal coupling between gauge potentials $\left(A_{\mu}\right.$ and $\left.A_{\mu}^{5}\right)$ and currents is also valid for nonrelativistic fermions [71], the interaction terms are $A_{\mu} j^{\mu}$ and $A_{\mu}^{5} j^{5 \mu}$. Therefore $m_{\mu} j^{\mu}$ and $b_{\mu} j^{5 \mu}$ can be replaced by using Eqs. (24) and (25). For example, $-\int b_{\mu} j^{\mu}=\int\left(b_{\mu} x^{\mu}\right) \partial_{\mu} j^{\mu}$ and the corresponding 
TABLE II. Topological responses in both single- and multi-Weyl semimetals. The "Form" column is valid for both single- and multi-Weyl semimetals in Eq. (1); the "References" column is for the corresponding references on single-Weyl semimetals. (We adopt the Gauss unit in this table so as to connect with results in other references.)

\begin{tabular}{lcc}
\hline \hline Name & Form & References \\
\hline phonon Hall viscosity & $S_{\text {eff }}=-i \frac{n e^{2}}{4 \pi^{2} \hbar} \int d^{d} x \epsilon^{\mu \nu \rho \sigma} b_{\mu} A_{v}^{5} \partial_{\rho} A_{\sigma}^{5}$ & {$[54]$} \\
charge density & $j^{0}=\frac{n e^{2}}{2 \pi^{2} \hbar^{2}} \mathbf{b} \cdot \mathbf{B}$ & {$[10]$} \\
chiral magnetic effect & $\mathbf{j}=\frac{n e^{2}}{2 \pi^{2} \hbar^{2}} b_{0} \mathbf{B}$ & {$[7,8]$} \\
AHE & $\mathbf{j}=-\frac{n e^{2}}{2 \pi^{2} \hbar^{2}} \mathbf{b} \times \mathbf{E}$ & {$[21,22]$} \\
chiral pseudomagnetic effect & $\mathbf{j}=\frac{n e^{2}}{2 \pi^{2} \hbar^{2}} m_{0} \mathbf{B}_{5}$ & {$[11,56-58]$} \\
axial charge density & $j^{50}=\frac{n e^{2}}{2 \pi^{2} \hbar^{2}} \mathbf{b} \cdot \mathbf{B}_{5}$ & \\
chiral separation effect & $\mathbf{j}^{5}=\frac{n e^{2}}{2 \pi^{2} \hbar^{2}} m_{0} \mathbf{B}$ & $\mathbf{j}^{5}=\frac{n e^{2}}{2 \pi^{2} \hbar^{2}} b_{0} \mathbf{B}_{5}$ \\
axial pseudoseparation effect & $\mathbf{j}^{\mathbf{5}}=-\frac{n e^{2}}{2 \pi^{2} \hbar^{2}} \mathbf{b} \times \mathbf{E}_{5}$ & this paper \\
anomalous axial Hall effect & this paper \\
\hline \hline
\end{tabular}

action is

$$
\begin{aligned}
S_{\mathrm{eff}}= & -i \frac{n e^{2}}{4 \pi^{2}} \epsilon^{\mu \nu \rho \sigma} \\
& \times \int\left[b_{\mu}\left(A_{\nu} \partial_{\rho} A_{\sigma}+A_{\nu}^{5} \partial_{\rho} A_{\sigma}^{5}\right)+2 m_{\mu} A_{\nu} \partial_{\rho} A_{\sigma}^{5}\right],
\end{aligned}
$$

which captures the topological responses associated with $b_{\mu}$ and $m_{\mu}$. For $n=1$, the second term in Eq. (28) is nothing but the phonon Hall viscosity proposed in single-Weyl semimetals [54]. The topological responses to the fields can be obtained by varying the effective action $S_{\text {eff }}$ with respect to $A_{\mu}$ and $A_{\mu}^{5}$ :

$$
j^{\mu}=\frac{n e^{2}}{2 \pi^{2}} \epsilon^{\mu \nu \rho \sigma}\left(b_{\nu} \partial_{\rho} A_{\sigma}+m_{\nu} \partial_{\rho} A_{\sigma}^{5}\right)
$$

and

$$
j^{5 \mu}=\frac{n e^{2}}{2 \pi^{2}} \epsilon^{\mu \nu \rho \sigma}\left(b_{\nu} \partial_{\rho} A_{\sigma}^{5}+m_{\nu} \partial_{\rho} A_{\sigma}\right) .
$$

Note that their explicit expressions in terms of $\mathbf{E}, \mathbf{B}, \mathbf{E}_{5}$, and $\mathbf{B}_{5}$ are listed in Table II. It is clear that the axial currents in Eq. (30) can be obtained from $j^{\mu}$ by interchanging $A_{\sigma}$ with $A_{\sigma}^{5}$, and vice versa. Alternatively, the current in Eq. (29) can be written as a sum of a polarization current and a magnetization current (the details are given in Appendix D):

$$
\mathbf{j}=\partial_{t} \mathbf{P}+\nabla \times \mathbf{M},
$$

where the polarization vector $\mathbf{P}$ and the magnetization vector $\mathbf{M}$ are defined by

$$
\mathbf{P}=\frac{n e^{2}}{2 \pi^{2}}\left[\left(b_{\alpha} x^{\alpha}\right) \mathbf{B}+\left(m_{\alpha} x^{\alpha}\right) \mathbf{B}_{5}\right]
$$

and

$$
\mathbf{M}=\frac{n e^{2}}{2 \pi^{2}}\left[\left(b_{\alpha} x^{\alpha}\right) \mathbf{E}+\left(m_{\alpha} x^{\alpha}\right) \mathbf{E}_{5}\right],
$$

respectively. For $n=1$, setting the axion field $b_{\alpha} x^{\alpha}=-\pi / 2$ and the axial electromagnetic fields $\mathbf{B}_{5}=0$ and $\mathbf{E}_{5}=0$, one yields $\mathbf{P}=-e^{2} \mathbf{B} / 4 \pi$, which is exactly the topological magnetoelectric effect [72]. Specifically, the charge density and the current density can be recast as

$$
j^{0}=\frac{n e^{2}}{2 \pi^{2}}\left(\mathbf{b} \cdot \mathbf{B}+\mathbf{m} \cdot \mathbf{B}_{5}\right)
$$

and

$$
\mathbf{j}=\frac{n e^{2}}{2 \pi^{2}}\left(b_{0} \mathbf{B}+m_{0} \mathbf{B}_{5}-\mathbf{b} \times \mathbf{E}-\mathbf{m} \times \mathbf{E}_{5}\right) .
$$

The axial charge and current densities are given as

$$
j^{50}=\frac{n e^{2}}{2 \pi^{2}}\left(\mathbf{b} \cdot \mathbf{B}_{5}+\mathbf{m} \cdot \mathbf{B}\right)
$$

and

$$
\mathbf{j}^{\mathbf{5}}=\frac{n e^{2}}{2 \pi^{2}}\left(m_{0} \mathbf{B}+b_{0} \mathbf{B}_{5}-\mathbf{m} \times \mathbf{E}-\mathbf{b} \times \mathbf{E}_{5}\right) .
$$

The first term in Eq. (35) is the celebrated chiral magnetic effect [7]. The second term relates to the newly predicted chiral pseudomagnetic effect [11,56-58], which can enhance the magnetoconductivity [57]. The third term in Eq. (35) is the AHE [73]. It is known that the two-dimensional integer Hall conductance (with a unit normal vector $\hat{\mathbf{k}}$ ) can be written as $\mathbf{j}=-\frac{n e^{2}}{2 \pi^{2}} \hat{\mathbf{k}} \times \mathbf{E}$. Therefore, from the viewpoint of the anomalous Hall conductivity, $\mathbf{j}=-\frac{n e^{2}}{2 \pi^{2}} \mathbf{b} \times \mathbf{E}$ indicates the possible connection between the Weyl semimetals without time reversal symmetry and the two-dimensional Chern insulators.

The first term in Eq. (37) is known as the chiral separation effect in quantum chromodynamics [67,68] and the analog of the valley current in valleytronics [11]. It can also be understood as follows: under an external magnetic field, the right-handed fermions and the left-handed fermions move parallel to and antiparallel to the magnetic field, due to opposite chirality $[11,26]$. To the best of our knowledge, the second term is firstly derived in this paper and needs a nonzero chiral chemical potential $b_{0}$. Physically, the axial magnetic field $\mathbf{B}_{5}$ initially induces an electric current for both left-handed fermions and right-handed fermions [11]. An extra negative sign from $b_{0}$ leads to an axial current. It is our second main result. The final term in Eq. (37) can be regarded as a cousin of AHE. It originates from the fact that $\mathbf{E}_{5}$ couples with opposite signs to the left- and right-handed Weyl fermions. 


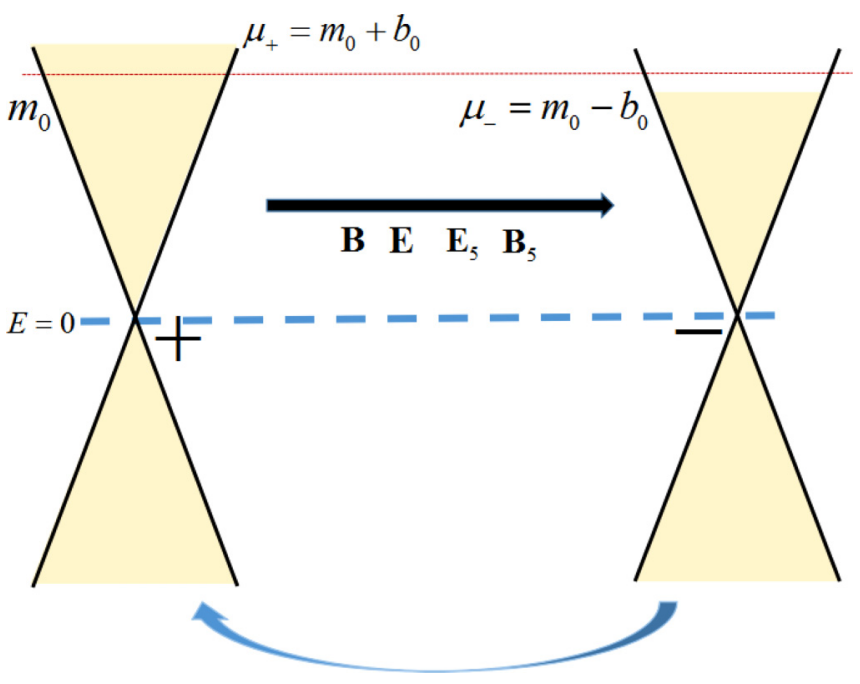

FIG. 1. Chiral anomaly in the presence of both the external electromagnetic fields $\mathbf{E}$ and $\mathbf{B}$ as well as the axial fields $\mathbf{E}_{5}$ and $\mathbf{B}_{5} . \pm$ refer to the chirality of Weyl nodes. The blue arrow denotes for the charge transfer between two Weyl nodes with opposite chirality.

The occurrence of all the terms linear in $\mathbf{m}$ above requires the nonzero sum of center of momentum of all Weyl nodes.

All the terms in Eq. (29) and Eq. (30) can also be obtained in a physically intuitive way, i.e. the energy balance argument [26] and the force balance argument. We first focus on physics near Weyl node + with an effective chemical potential $m_{0}+$ $b_{0}$. When both electromagnetic and axial electromagnetic fields are turned on, Eq. (26) seemingly implies that there are quasiparticles with energy $m_{0}+b_{0}$ created from the Dirac sea. On the other hand, the quasiparticles with an opposite chirality annihilate (see Fig. 1). Therefore we generalize the elegant energy balance argument [26] to the present case with both electromagnetic fields and axial fields:

$$
\begin{aligned}
\mathbf{j}_{+} & \cdot \mathbf{E}_{+}+\mathbf{j}_{-} \cdot \mathbf{E}_{-} \\
& =\frac{n e^{2}}{4 \pi^{2}}\left[\left(m_{0}+b_{0}\right) \mathbf{E}_{+} \cdot \mathbf{B}_{+}-\left(m_{0}-b_{0}\right) \mathbf{E}_{-} \cdot \mathbf{B}_{-}\right] .
\end{aligned}
$$

Physically, the first line is the energy extracted from external fields and the second line equals the energy due to charge pumping. Note that the left-hand side of Eq. (38) equals $\mathbf{j} \cdot \mathbf{E}+$ $\mathbf{j}^{5} \cdot \mathbf{E}_{5}$ with the total current $\mathbf{j}=\mathbf{j}_{+}+\mathbf{j}_{-}$and the axial current $\mathbf{j}^{5}=\mathbf{j}_{+}-\mathbf{j}_{-}$, which implies that the axial current couples to the axial electric field and costs energy.

Phenomenologically, because of the nonzero averaged momentum of quasiparticles near Weyl nodes \pm , energy transfer in Eq. (38) must accompany with transfer of momentum of quasiparticles (see Fig. 1). The total forces exerting on quasiparticles should vanish, including the forces due to momentum transfer, the electric forces and the Lorentz forces, that is, the force balance condition

$$
\sum_{s= \pm 1}\left(j_{s}^{0} \mathbf{E}_{s}+\mathbf{j}_{s} \times \mathbf{B}_{s}\right)=\frac{n e^{2}}{4 \pi^{2}} \sum_{s= \pm 1} s(\mathbf{m}+s \mathbf{b}) \mathbf{E}_{s} \cdot \mathbf{B}_{s} .
$$

Since both the energy balance argument in Eq. (38) and the force balance condition in Eq. (39) are valid for arbitrary external fields, one can employ vector analysis to obtain the corresponding charge density and the current density, which exactly coincide with those in Eqs. (34)-(37).

Note that Eq. (29) does not obey the continuity equations in Eqs. (24) and (25). To restore the continuity equations above, other terms are needed to be included. Since $A_{v}^{5}$ is observable but $A_{\mu}$ not [54], we write these extra terms as

$$
\delta j^{\mu}=\frac{n e^{3}}{2 \pi^{2}} \epsilon^{\mu \nu \rho \sigma} A_{\nu}^{5} \partial_{\rho} A_{\sigma},
$$

which refers to a Chern-Simons contribution to the current density in Eq. (35) [70,74]. Its spatial components can be recast as

$$
\delta \mathbf{j}=\frac{n e^{3}}{2 \pi^{2}}\left(A_{0}^{5} \mathbf{B}-\mathbf{A}^{5} \times \mathbf{E}\right)
$$

while the temporal component is given as $j^{0}=\frac{n e^{3}}{2 \pi^{2}} \mathbf{A}^{5} \cdot \mathbf{B}$. The first term in Eq. (41) is the strain-induced chiral magnetic effect [66], whereas the second term can be dubbed as the strain-induced AHE. This strain-induced AHE can be understood by the following argument: the strain fields achieved from stretching or compressing the sample would alter the crystal constants in some direction. Consequently, the distance between the Weyl nodes with opposite chirality in momentum space is effectively changed by $\mathbf{A}^{5}$, leading to a modification to the anomalous Hall current. The topological Chern-Simons terms in Eq. (40) are regarded as a ground-state current coming from the carriers far from the Fermi surface, which are not well described by the effective Hamiltonian near each Weyl node $[14,53]$. This scenario has recently been used to construct the semiclassical chiral kinetic theory to investigate plasmons in Weyl materials [62]. It should be noted that, in confined systems with boundaries (nanowires or thin films), the local nonconservation of the electric current in Eq. (29) is attributed to a charge pumping between the bulk and the surface [58]. Thus there is no global charge nonconservation.

Before closing this section, let us discuss the realization of the strain-induced AHE in double-Weyl semimetals (the details are given in Appendix C). For the sake of simplicity, we assume that $\mathbf{b}$ is along the $z$ direction in this case, that is, $\mathbf{b}=b_{3} \hat{z}$. A longitudinal sound wave with frequency $\omega$ along $z$ direction can produce a displacement field, $\mathbf{u}=u_{0} \sin (q z-\omega t) \hat{z}$, which gives rise to $\mathbf{A}^{5}=$ $\left(0,0,-\frac{1}{e a} \cot \left(a b_{3}\right) u_{0} q \cos (q z-\omega t)\right)$ ( $a$ is the lattice constant) [58]. In the limit $a b_{3} \ll 1$, one approximates $\cot \left(a b_{3}\right) \simeq$ $1 / a b_{3}$ and then gets the anomalous Hall conductivity as

$$
\sigma_{y x}=\frac{e^{2} u_{0} q}{\pi^{2} a^{2} b_{3}} \cos (q z-\omega t) .
$$

The estimation the corresponding coefficients is carried out as follows: $b_{3} \sim 2 \pi / \chi a, u_{0} \sim 10^{-2} a, c_{s} \sim 2.3 \times 10^{3} \mathrm{~m} / \mathrm{s}$ (sound speed) and $\lambda_{s} \sim 11 \times 10^{-6} \mathrm{~m}$ (sound wavelength) $[58,75]$. The wave vector is thus of order $q \sim 2 \pi / \lambda_{s}$ and the axial vector potential $\mathbf{A}^{5} \sim 0.01 \chi / e \lambda_{s}$. Therefore the Hall conductance is $\sigma_{y x} \sim \frac{e^{2} \chi}{200 \pi^{2} \lambda_{s}}$. The ratio between this one and the conventional anomalous Hall conductance is of order: $0.01 \chi^{2} a / \lambda_{s}$. Since the lattice constant is of order $10^{-10} \mathrm{~m}$ and $\lambda_{s}$ of order $10^{-5} \mathrm{~m}$, this effect is comparable with the conventional one if $\chi$ is of order $10^{3}$ or larger. 


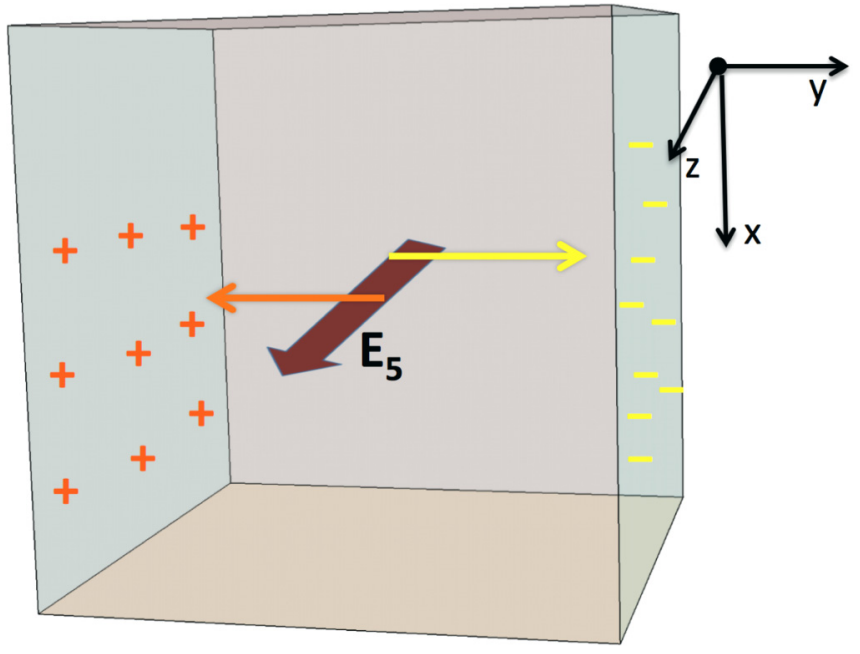

FIG. 2. Schematic figure showing the anomalous axial Hall effect in Table II driven by an axial electric field $\mathbf{E}_{5}$ and the resulting dynamical chirality accumulation at the surfaces perpendicular to this axial current.

A finite anomalous axial Hall effect in Table II requires an axial electric field that deviates from the direction of vector $\mathbf{b}=\left(b_{1}, 0, b_{3}\right)$. Following the procedure above, one immediately gets $\mathbf{E}_{5}(t)=\frac{1}{e a} \cot \left(a b_{3}\right) u_{0} q \omega \sin (q z-\omega t) \hat{z}$. Thus the magnitude of the anomalous axial Hall current in the $y$ direction in the limit $a b_{3} \ll 1$ becomes

$$
j^{5 y}=-\frac{e u_{0} q \omega b_{1}}{\pi^{2} a^{2} b_{3}} \sin (q z-\omega t)
$$

which leads to the chirality accumulation at the surfaces perpendicular to the $y$ direction, as shown in Fig. 2. It is our third main result. This effect can be seen as the three-dimensional counterpart of the valley Hall effect in graphenelike systems [76], in which electrons in different valleys follow in opposite directions perpendicular to the electric field then accumulate near different boundaries of systems. It is instructive to compare the chirality accumulation created by an axial electric field with that induced by a magnetic field through the chiral separation effect [11]. First, the former occurs at the surfaces perpendicular to the cross product of $\mathbf{b} \times \mathbf{E}_{5}$, while the latter is at the surfaces perpendicular to the magnetic field. Second, the magnitude of the one due to the axial electric field is independent of the chemical potential, whereas that of the magnetic field-induced one is linear in the chemical potential.

Now we turn to estimate this chirality accumulation by considering a half-infinity large system locate at $y \geqslant 0$ with open boundary at $y=0$. Due to the translational symmetry in the $x$ direction, one thus assumes that the chirality density is independent of $x$. The continuity equation of the axial current near the surface is modified to

$$
\partial_{t} j^{50}(y, z, t)=-\frac{1}{\tau_{c}} j^{50}(y, z, t)-\nabla \cdot \mathbf{j}^{5}(y, z, t)
$$

where $\tau_{c}$ is the relaxation time of the chirality density near the surface and $\mathbf{j}^{5}$ comes from the diffusion law and the anomalous axial Hall current

$$
j^{5 y}=-D_{5} \partial_{y} j^{50}(y, z, t)-\frac{e u_{0} q \omega b_{1}}{\pi^{2} a^{2} b_{3}} \sin (q z-\omega t),
$$

where $D_{5}$ is the diffusion parameter. We impose the boundary condition that $j^{5 y}$ vanishes at $y=0$. By combining these two equations together, one can find

$$
\partial_{t} j^{50}(y, z, t)=-\frac{1}{\tau_{c}} j^{50}(y, z, t)+D_{5} \partial_{y}^{2} j^{50}(y, z, t),
$$

which is exactly a diffusion function of the chirality density with the relaxation time $\tau_{c}$. For simplicity, we consider the limit of $\omega \ll \tau_{c}^{-1}$ and take following ansatz:

$$
j^{50}(y, z, t)=\operatorname{Im}\left\{\rho_{a}(y) \exp [i(q z-\omega t)]\right\} .
$$

Note that our ansatz is valid in the limit of $\operatorname{Im} \rho_{a}(y) \ll$ $\operatorname{Re} \rho_{a}(y)$. Inserting Eq. (47) into Eq. (46) leads to

$$
\rho_{a}(y)=\frac{e u_{0} q \omega b_{1}}{\pi^{2} a^{2} b_{3} \lambda D_{5}} \exp (-\lambda y),
$$

where $\lambda \equiv \sqrt{\left(-i \omega+1 / \tau_{c}\right) / D_{5}}$ and the factor before $\exp (-\lambda y)$ is determined by the boundary conditions: $\left.\rho_{a}(y)\right|_{y \rightarrow+\infty}=0$ and $\left.j^{5 y}(y, z, t)\right|_{y=0}=0$. In the limit $\omega \ll$ $\tau_{c}^{-1}, j^{50}(y, z, t)$ can be further simplified as

$$
j^{50}(y, z, t)=\frac{e u_{0} q \omega b_{1} \sqrt{D_{5}}}{\pi^{2} a^{2} b_{3} \sqrt{\tau_{c}}} e^{\frac{-y}{\sqrt{D_{5} \tau_{c}}}} \sin (q z-\omega t),
$$

which suggests that the chirality is confined to a narrow region of order $\delta y \sim \sqrt{D_{5} \tau_{c}}$.

In reality, there are three characteristic timescales that are associated with the detection of the chirality accumulation induced by an alternating axial electric field: the period of the axial electric field $2 \pi / \omega, \tau_{c}$, and the period of the probe light $\tau_{p}$. The period of the modern ultrafast probe light can be of the order of a femtosecond, while the frequency of the axial electric field is typically several hundred megahertz. $\tau_{c}$, a material-dependent parameter, might be dominated by the internode relaxation time $\tau_{v}$, which has been recently evaluated from the measurement of magnetotransport in zirconium pentatelluride, $\tau_{v}^{-1} \sim$ terahertz [34]. The observation of the chirality accumulation requires these characteristic times satisfy the condition: $\omega^{-1} \gg \tau_{p}$ and $\tau_{c} \gg \tau_{p}$. Due to the alternating nature of the axial electric field, there is no net stationary chirality accumulation during a long time interval.

\section{DISCUSSIONS AND CONCLUSIONS}

We perform detailed derivations of the equations of the chiral anomaly for multi-Weyl semimetals by using the Fujikawa's method and emphasize several manifestations of the broken Lorentz symmetry due to their nonlinear nature of energy dispersions. It has also been pointed out that the equations of the chiral anomaly for multi-Weyl semimetals differ from those for single-Weyl semimetals by the higher winding number $n$. It can be understood from the intuitive picture of the chiral anomaly in the language of the chiral Landau levels.

Compared with single-Weyl semimetals, the transport properties of multi-Weyl semimetals are modified by higher monopole charges or winding numbers. The axial vector 
potential contributes a modification to the anomalous Hall conductivity. Meanwhile, both the axial electric and the axial magnetic fields make contributions to the axial current. These axial currents may produce the chirality accumulation at the surfaces of a finite-size sample even in the absence of external magnetic fields. Our nonmagnetic mechanism of the dynamical chirality accumulation may possess great advantages in the application of Weyl semimetals in promising valleytronics $[76,77]$. On the other hand, the chirality accumulation in Weyl semimetals induced by a magnetic field through the chiral separation effect is difficult to identify unambiguously in circular dichroism spectroscopy. The impacts of impurities scattering and realistic boundary conditions of Weyl semimetals on a chirality accumulation are also critical issues for both the experimental detection and potential applications.

In addition, many topological responses are summarized in Table II, such as AHE, chiral magnetic effect, chiral pseudomagnetic effect and chiral separation effect, axial pseudoseparation effect and anomalous axial Hall effect. Finally, it should be noted that the realizations of general axial gauge fields in multi-Weyl semimetals depend on the specific properties of materials under consideration, which deserve further study in the future.

\section{ACKNOWLEDGMENTS}

The authors would like to thank Xiao Li, Dmitry Pikulin, Min-Fong Yang, and Song-Bo Zhang for helpful discussions and comments. We also thank Kai-Liang Huang for preparing the figures. This work was supported by the Research Grants Council, University Grants Committee, Hong Kong under Grants No. 17301116 and No. C6026-16W.

\section{APPENDIX A: JACOBIAN FOR CHIRAL TRANSFORMATION}

In this part, we provide detailed derivations of the Jacobian under an infinitesimal chiral transformation. For convenience, we focus on double-Weyl semimetals and then generalize to multi-Weyl semimetals. We also set $e=1$ hereafter.

\section{Model for multi-Weyl semimetals}

The effective Hamiltonian for multi-Weyl semimetals with a pair of Weyl nodes reads

$$
H_{s}=s\left[\mathbf{p}_{3} \sigma^{3}+w^{-1}\left(\mathbf{p}_{+}^{n} \sigma^{-}+\mathbf{p}_{-}^{n} \sigma^{+}\right)\right],
$$

where $n=2$ is the topological charge, $s= \pm 1$ are the chirality of Weyl nodes, $p_{ \pm}=\left(p_{1} \pm i p_{2}\right) / \sqrt{2}$ and $\sigma^{ \pm}=$ $\left(\sigma^{1} \pm i \sigma^{2}\right) / \sqrt{2}$. Consequently, we have

$$
\left\{\sigma^{3}, \sigma^{ \pm}\right\}=\left\{\sigma^{+}, \sigma^{+}\right\}=\left\{\sigma^{-}, \sigma^{-}\right\}=0
$$

and

$$
\left\{\sigma^{+}, \sigma^{-}\right\}=\left\{\sigma^{-}, \sigma^{+}\right\}=\left\{\sigma^{3}, \sigma^{3}\right\}=2 .
$$

The corresponding Lagrangian density is given as

$$
\begin{aligned}
\mathcal{L} & =\Sigma_{s} \Psi_{s}^{\dagger}\left(p_{0}-H_{s}\right) \Psi_{s} \\
& =\bar{\Psi}\left[p_{0} \gamma^{0}+p_{3} \gamma^{3}+w^{-1}\left(p_{+}^{n} \gamma^{+}+p_{-}^{n} \gamma^{-}\right)\right] \Psi
\end{aligned}
$$

with $\Psi=\left(\Psi_{+}, \Psi_{-}\right)^{T}, \bar{\Psi}=\Psi^{\dagger} \gamma^{0}, \gamma^{ \pm}=\left(\gamma^{1} \mp i \gamma^{2}\right) / \sqrt{2}$ and $\left(\gamma^{ \pm}\right)^{\dagger}=-\gamma^{\mp}$. For later convenience, we shall turn to the Euclidean spacetime.

\section{The Euclidean-spacetime action}

Performing a Wick's rotation, i.e., $p_{0}=i \omega$, one gets the partition function

$$
\begin{aligned}
Z= & \int \mathcal{D}[\bar{\Psi}, \Psi] \exp \left\{i \int d^{d} x_{E}\right. \\
& \left.\times \bar{\Psi}\left[\omega \gamma_{E}^{0}+p_{3} \gamma_{E}^{3}+w^{-1}\left(p_{+}^{n} \gamma_{E}^{+}+p_{-}^{n} \gamma_{E}^{-}\right)\right] \Psi\right\},
\end{aligned}
$$

where $\gamma_{E}^{0}=\gamma^{0}, \quad \gamma_{E}^{3}=-i \gamma^{3}, \quad \gamma_{E}^{ \pm}=-i \gamma^{ \pm}, \quad$ satisfying $\left\{\gamma_{E}^{\alpha}, \gamma_{E}^{\beta}\right\}=2 g_{E}^{\alpha \beta}$. The metric tensor is given as

$$
g_{E}^{\alpha \beta}=\left(\begin{array}{llll}
1 & 0 & 0 & 0 \\
0 & 0 & 1 & 0 \\
0 & 1 & 0 & 0 \\
0 & 0 & 0 & 1
\end{array}\right)
$$

with $\alpha, \beta=0,+,-, 3$. Therefore $i \not D^{n}=i D_{\alpha}^{n} \gamma_{E}^{\alpha}$ is a Hermitian operator with components

$$
\begin{aligned}
& i D_{0}^{n}(A)=\omega-A_{0}, \\
& i D_{ \pm}^{n}(A)=\left(p_{ \pm}-A_{ \pm}\right)^{n} / w, \\
& i D_{3}^{n}(A)=p_{3}-A_{3} .
\end{aligned}
$$

\section{Fujikawa's method and chiral transformation}

Following the standard and lengthy derivation [47,69], one gets the measure for a global chiral transformation $\Psi^{\prime}=$ $\exp \left(i \beta \gamma^{5}\right) \Psi$ as

$$
\mathcal{D} \bar{\Psi}^{\prime} \mathcal{D} \Psi^{\prime}=J[\beta] \mathcal{D} \bar{\Psi} \mathcal{D} \Psi
$$

where

$$
J[\beta]=\exp \left[-2 i \beta \int \lim _{M \rightarrow \infty} \operatorname{tr} \gamma^{5} e^{-\left|i \not^{n}\right|^{2} / M^{2}} \delta(x-x)\right]
$$

with

$$
\left|i \not D^{n}\right|^{2}=g_{E}^{\alpha \beta}\left(i D_{\alpha}^{n=2}\right)\left(i D_{\beta}^{n=2}\right)+\frac{1}{2} \gamma_{E}^{\alpha} \gamma_{E}^{\beta}\left[i D_{\alpha}^{n=2}, i D_{\beta}^{n=2}\right] .
$$

Defining $4 \epsilon_{E}^{\alpha_{1} \alpha_{2} \alpha_{3} \alpha_{4}}=\operatorname{tr}\left(\gamma^{5} \gamma_{E}^{\alpha_{1}} \gamma_{E}^{\alpha_{2}} \gamma_{E}^{\alpha_{3}} \gamma_{E}^{\alpha_{4}}\right)$ and performing a Fourier transformation

$$
\begin{aligned}
\lim _{x \rightarrow y} e^{-\left|i D^{n=2}\right|^{2} / M^{2}} \delta(x-y)= & \int \frac{d^{d} k}{(2 \pi)^{d}} e^{-\left|i D^{n=2}\right|^{2} / M^{2}} \\
& (p \rightarrow p-k),
\end{aligned}
$$


we get the Jacobian as

$$
\frac{\ln [J]}{-2 i \beta}=\lim _{M \rightarrow \infty} \int \frac{d^{d} k}{(2 \pi)^{d}} \epsilon_{E}^{\alpha_{1} \alpha_{2} \alpha_{3} \alpha_{4}} e^{-\frac{k_{0}^{2}+k_{3}^{2}+\left(k_{1}^{2}+k_{2}^{2}\right)^{2} / 2 w^{2}}{M^{2}}} \frac{\left[i D_{\alpha_{1}}^{n=2}, i D_{\alpha_{2}}^{n=2}\right]\left[i D_{\alpha_{3}}^{n=2}, i D_{\alpha_{4}}^{n=2}\right]}{4 M^{4}} .
$$

Note that other terms vanish due to the infinitely large $M$ and the trace of gamma matrices. The factor of $\exp \left\{-\left[k_{0}^{2}+k_{3}^{2}+\left(k_{1}^{2}+k_{2}^{2}\right)^{2} / 2 w^{2}\right] / M^{2}\right\}$ comes from $g_{E}^{\alpha \beta}\left(i D_{\alpha}^{n=2}\right)\left(i D_{\beta}^{n=2}\right)$. As for the commutators, they are given as

$$
\begin{aligned}
w^{2}\left[i D_{0}^{n=2}, i D_{3}^{n=2}\right]\left[i D_{-}^{n=2}, i D_{+}^{n=2}\right]= & -i F_{03}\left\{i \partial_{+} \partial_{-} F_{-+}+2\left[\left(\partial_{-} F_{-+}\right) i D_{+}+\left(\partial_{+} F_{-+}\right) i D_{-}\right]+2 i F_{-+}\left\{D_{+}, D_{-}\right\}\right\} \\
w^{2}\left[i D_{-}^{n=2}, i D_{+}^{n=2}\right]\left[i D_{0}^{n=2}, i D_{3}^{n=2}\right]= & \left(\partial_{+} \partial_{-} F_{-+}\right) F_{03}+\left[2\left(\partial_{-} F_{-+}\right)\left(\partial_{+} F_{03}\right)+2\left(\partial_{+} F_{-+}\right)\left(\partial_{-} F_{03}\right)\right] \\
& +\left[4 F_{-+}\left(\partial_{+} \partial_{-} F_{03}\right)+2 F_{-+} F_{03}\left\{D_{+}, D_{-}\right\}\right] \\
w^{2}\left[i D_{0}^{n=2}, i D_{+}^{n=2}\right]\left[i D_{3}^{n=2}, i D_{-}^{n=2}\right]= & \left(\partial_{+} F_{0+}\right)\left(\partial_{-} F_{3-}\right)+2 F_{0+}\left(\partial_{+} \partial_{-} F_{3-}\right)+4 F_{0+} F_{3-} D_{+} D_{-} .
\end{aligned}
$$

Note that one can exchange subscripts + and - to obtain all the rest terms.

It is clear that the product of commutators contain not only functions, but also operators, e.g., $\partial F_{\alpha \beta} D_{ \pm}$and $F_{\alpha \beta} F_{\mu \nu}\left|D_{ \pm}\right|^{2}$. This is due to the quadratic dispersion of double-Weyl semimetals. However, we shall show that only terms containing $\left|D_{ \pm}\right|^{2}$ survive in the limit of $M \rightarrow \infty$. We first focus on the part contributed by $\left|D_{ \pm}\right|^{2}$ :

$$
\begin{aligned}
\frac{\ln J}{-2 i \beta}= & \frac{1}{2}\left(4 \epsilon_{E}^{\alpha_{1} \alpha_{2} \alpha_{3} \alpha_{4}}\right) \lim _{M \rightarrow \infty} \int \frac{d^{d} k}{(2 \pi)^{d}} M^{3} w e^{-\left[k_{0}^{2}+k_{3}^{2}+\left(k_{1}^{2}+k_{2}^{2}\right)^{2} / 2\right]} \\
& \times \frac{M w}{4 M^{4} w^{2}}\left[-4 F_{\alpha_{1} \alpha_{2}} F_{\alpha_{3} \alpha_{4}} \frac{\left(k_{1}+A_{1} M^{-1 / 2} w^{-1 / 2}\right)^{2}+\left(k_{2}+A_{2} M^{-1 / 2} w^{-1 / 2}\right)^{2}}{2}\right] \\
= & \frac{4 \epsilon_{E}^{\alpha_{1} \alpha_{2} \alpha_{3} \alpha_{4}}}{8} \lim _{M \rightarrow \infty} \int \frac{d^{d} k}{(2 \pi)^{d}}\left[-2 F_{\alpha_{1} \alpha_{2}} F_{\alpha_{3} \alpha_{4}}\left(k_{1}^{2}+k_{2}^{2}\right)\right] e^{-\left[k_{0}^{2}+k_{3}^{2}+\left(k_{1}^{2}+k_{2}^{2}\right)^{2} / 2\right]} \\
= & \frac{1}{16 \pi^{2}} \epsilon^{\mu \nu \alpha \beta} F_{\mu \nu} F_{\alpha \beta},
\end{aligned}
$$

where we have rescaled $k_{0,3} \rightarrow M k_{0,3}$ and $k_{1,2} \rightarrow \sqrt{w M} k_{1,2}$. For $\partial F_{\alpha_{1} \alpha_{2}} \partial F_{\alpha_{3} \alpha_{4}}$, the integral is proportional to $\frac{1}{M w}$, and $\frac{1}{\sqrt{M w}}$ for $F_{\alpha_{1} \alpha_{2}} \partial F_{\alpha_{3} \alpha_{4}}$. Therefore only $F_{\alpha_{1} \alpha_{2}} F_{\alpha_{3} \alpha_{4}}$ survives in the limit $M \rightarrow \infty$. Note that for multi-Weyl semimetals with winding number $n, k_{\mu}$ are scaled as $k_{0,3} \rightarrow M k_{0,3}, k_{1,2} \rightarrow M^{1 / n} k_{1,2}$ ( $w$ is neglected). Thus the coefficient for $F_{\alpha_{1} \alpha_{2}} F_{\alpha_{3} \alpha_{4}}$ is proportional to $M^{2+2 / n} M^{-4} M^{\frac{2 n-2}{n}}=M^{0}$, where $M^{2+2 / n}, M^{-4}$ and $M^{\frac{2 n-2}{n}}$ come from rescaling of $d^{4} k$, the exponent and $\left|D_{ \pm}\right|^{2 n-2}$, respectively. All other terms are suppressed by taking the limit of $M \rightarrow \infty$. Hence the Jacobian for multi-Weyl semimetals with winding number $n$ is

$$
\begin{aligned}
\frac{\ln J}{-2 i \beta} & =\frac{1}{2}\left(4 \epsilon_{E}^{\alpha_{1} \alpha_{2} \alpha_{3} \alpha_{4}}\right) \lim _{M \rightarrow \infty} \int \frac{d^{d} k}{(2 \pi)^{d}} e^{-\left\{k_{0}^{2}+k_{3}^{2}+2\left[\left(k_{1}^{2}+k_{2}^{2}\right) / 2\right]^{n}\right\}} \frac{1}{4}\left\{-n^{2} F_{\alpha_{1} \alpha_{2}} F_{\alpha_{3} \alpha_{4}}\left[\left(k_{1}^{2}+k_{2}^{2}\right) / 2\right]^{n-1}\right\} \\
& =\frac{n}{32 \pi^{2}} \epsilon^{\mu \nu \alpha \beta} F_{\mu \nu} F_{\alpha \beta} .
\end{aligned}
$$

Note that the coefficient $n^{2}$ in the second line comes from the commutator: $\left[i D_{+}^{n}, i D_{-}^{n}\right]$ or $\left[i D_{-}^{n}, i D_{+}^{n}\right]$.

\section{APPENDIX B: EFFECTIVE ACTION IN THE ABSENCE OF AXIAL GAUGE FIELDS}

Now we shall derive the effective action for topological responses to electromagnetic fields with two different ways: Fujikawa's method and a perturbative approach. This enables us to visualize how the winding number manifests itself in the effective action. A chiral transformation is implemented as follows:

$$
\mathcal{L}=\bar{\Psi} e^{i b_{\mu} x^{\mu} \gamma^{5}}\left\{\gamma^{0}\left(p_{0}+b_{0} \gamma^{5}\right)+\gamma^{3}\left(p_{3}+b_{3} \gamma^{5}\right)+\frac{1}{w}\left[\gamma^{+}\left(p_{+}+b_{+} \gamma^{5}\right)^{n}+\gamma^{-}\left(p_{-}+b_{-} \gamma^{5}\right)^{n}\right]\right\} e^{i b_{\mu} x^{\mu} \gamma^{5}} \Psi
$$

where $b_{\mu} x^{\mu}$ is not an infinitesimal parameter. Therefore we iterate a sequence of infinitesimal chiral transformations: $\Psi \rightarrow$ $e^{i b_{\mu} x^{\mu} \gamma^{5} d s} \Psi$, with $d s$ an infinitesimal parameter. Hence, after a series of infinitesimal chiral transformations, the Lagrangian density becomes

$$
\mathcal{L}(s)=\bar{\Psi}\left\{\gamma^{0}\left[p_{0}+(1-s) b_{0} \gamma^{5}\right]+\gamma^{3}\left[p_{3}+(1-s) b_{3} \gamma^{5}\right]+\frac{1}{w} \gamma^{+}\left[p_{+}+(1-s) b_{+} \gamma^{5}\right]^{n}+\frac{1}{w} \gamma^{-}\left[p_{-}+(1-s) b_{-} \gamma^{5}\right]^{n}\right\} \Psi
$$


where $b_{\mu}$ is thus eliminated when $s=1$. Then we sum all these resulting Jacobians up. Because of Eq. (20), the effective action turns out to be

$$
\begin{aligned}
S_{\mathrm{eff}} & =i \frac{n}{16 \pi^{2}} \int d^{d} x \int_{0}^{1} d s\left(b_{\mu} x^{\mu}\right) \epsilon^{\mu \nu \rho \sigma}\left[F_{\mu \nu} F_{\rho \sigma}+F_{\mu \nu}^{5}(s) F_{\rho \sigma}^{5}(s)\right] \\
& =i \frac{n}{16 \pi^{2}} \int d^{d} x\left(b_{\mu} x^{\mu}\right) \epsilon^{\mu \nu \rho \sigma} F_{\mu \nu} F_{\rho \sigma},
\end{aligned}
$$

where $F_{\rho \sigma}^{5}(s)=(1-s)\left(\partial_{\rho} b_{\sigma}-\partial_{\sigma} b_{\rho}\right)=0$.

Let us resort to a perturbative approach to emphasize the role of topology. For simplicity, we set $b_{\mu}=-\delta_{\mu 3} b_{3}$. In the uniform and dc limit of external fields, the coefficient before $\epsilon^{3 \mu \nu \alpha} A_{\mu} \partial_{\nu} A_{\alpha}$ is given by

$$
\frac{i}{8 \pi^{2}} C=\frac{\epsilon^{3 \mu \nu \alpha}}{3 ! 2 !} \int \frac{d^{4} q}{(2 \pi)^{4}} \operatorname{tr}\left[\left(G \partial_{q_{\mu}} G^{-1}\right)\left(G \partial_{q_{v}} G^{-1}\right)\left(G \partial_{q_{\alpha}} G^{-1}\right)\right],
$$

where we have used Ward's identity

$$
-i \Gamma^{\mu}(p)=\partial_{p_{\mu}} G^{-1}(p)
$$

with $\Gamma^{\mu}(p)=\lim _{k \rightarrow 0} \Gamma^{\mu}(p+k, p) . \Gamma^{\mu}$ and $G(p)$ are the interacting vertex and real-time fermion Green's function, respectively. Note that this result is actually analogue to the Chern-Simons term in odd dimensional spacetime [78,79], for example, $S_{\mathrm{CS}}^{2+1} \propto$ $\left[\int\left(G d G^{-1}\right)^{3}\right] \int \epsilon^{\mu \nu \rho} A_{\mu} \partial_{\nu} A_{\rho}$ in $2+1$ dimension.

In addition, $C$ has a close relation with $N\left(\mathbf{p}_{3}\right)$ in Eq. (4),

$$
C=\int d p_{3} N\left(\mathbf{p}_{3}\right)=-2 n b,
$$

where $n$ originating from $N\left(\mathbf{p}_{3}\right)$ is the winding number. Hence, $n$ in Eq. (B3) is of topological nature.

\section{APPENDIX C: REALIZATION OF AXIAL GAUGE FIELDS IN DOUBLE-WEYL SEMIMETALS}

In this section, we shall construct the axial gauge fields in double-Weyl semimetals, which enable us to realize both the strain-induced anomalous Hall effect and the anomalous axial Hall effect in double-Weyl semimetals. We start with the following tight-binding Hamiltonian:

$$
\begin{aligned}
H= & {\left[t_{1} \cos \left(a k_{z}\right)-\Delta\right] \sigma^{3}+t\left\{\left[\sin \left(a k_{x}\right)-\alpha \sin \left(a k_{z}\right)\right]^{2}-\cos \left(a k_{y}\right)+1\right\} \sigma^{1} } \\
& +2 t\left[\sin \left(a k_{x}\right)-\alpha \sin \left(a k_{z}\right)\right] \sin \left(a k_{y}\right) \sigma^{2},
\end{aligned}
$$

where $a$ is the lattice constant, Pauli matrices $\sigma^{i}$ with $i=1,2,3$ have the same meanings as those in the main text, and a pair of Weyl nodes is located at $\pm \mathbf{b}$ in the three-dimensional Brillouin zone. The vector $\pm \mathbf{b}$ is given as

$$
\mathbf{b}=\left(b_{1}, 0, b_{3}\right),
$$

with $b_{1}=\alpha \sin \left(a b_{3}\right)$ and $b_{3}=a^{-1}\left|\arccos \left(\frac{\Delta}{t_{1}}\right)\right|$. The effective velocity along the $z$ direction is $v_{z}=s a t_{1} \sin \left(a b_{3}\right)$.

Now we apply a sound wave along the $z$ direction and get a displacement field

$$
\mathbf{u}=u_{0} \sin (q z-\omega t) \hat{z} .
$$

Consequently, the hopping constant along the $z$ direction is modified due to strain fields [54,59],

$$
t_{1} \sigma_{z} \rightarrow t_{1}\left(1-u_{33}\right) \sigma_{z},
$$

where $u_{33}=\partial_{z} u_{3}$. Hence the variation of the Hamiltonian is

$$
\delta H=-t_{1} u_{33} \cos \left(a k_{z}\right) \sigma_{z} \simeq-s t_{1} a \sin \left(a b_{3}\right)\left[\operatorname{seu}_{33} \frac{\cot \left(a b_{3}\right)}{a e}\right],
$$

where we have set $k_{z}=b_{3}$ in the last line.

Hence the Hamiltonian in the continuous limit is

$$
H_{s}=s\left\{\left(k_{z}+s b_{3}-s e A_{z}^{5}\right) \sigma^{3}+\left[\left(k_{x}+s b_{1}\right)^{2}-k_{y}^{2}\right] \sigma^{1}+2\left(k_{x}+s b_{1}\right) k_{y} \sigma^{2}\right\},
$$

where the effective velocity has been absorbed into the momentum, and the fermion field has been redefined: $\Psi=\left(\psi_{+}, \sigma^{3} \psi_{-}\right)$. The axial gauge field becomes

$$
A_{z}^{5}=-u_{33} \frac{\cot \left(a b_{3}\right)}{e a} \simeq-u_{33} \frac{1}{e a^{2} b_{3}}
$$

where $\simeq$ denotes for the limit of $a b_{3} \ll 1$. 


\section{APPENDIX D: POLARIZATION CURRENT AND MAGNETIZATION CURRENT}

The aim of this section is to show the current density in Eq. (29) can be expressed as a sum of a polarization current and a magnetization current. Let us first rewrite the current density as

$$
j^{\mu}=\frac{n e^{2}}{2 \pi^{2}} \epsilon^{\mu \nu \rho \sigma} \partial_{\nu}\left[\left(b_{\alpha} x^{\alpha}\right) \partial_{\rho} A_{\sigma}+\left(m_{\alpha} x^{\alpha}\right) \partial_{\rho} A_{\sigma}^{5}\right]
$$

Because of the antisymmetry property of the Levi-Civita symbol, the term of $\epsilon^{\mu \nu \rho \sigma} \partial_{\nu} \partial_{\rho} A_{\sigma}^{(5)}$ vanishes identically. Then the $i$ th component of the current density becomes

$$
j^{i}=\frac{n e^{2}}{2 \pi^{2}}\left\{\epsilon^{i 0 j k} \partial_{t}\left[\left(b_{\alpha} x^{\alpha}\right) \partial_{j} A_{k}+\left(m_{\alpha} x^{\alpha}\right) \partial_{j} A_{k}^{5}\right]+\epsilon^{i j 0 k} \partial_{j}\left[\left(b_{\alpha} x^{\alpha}\right) \partial_{t} A_{k}+\left(m_{\alpha} x^{\alpha}\right) \partial_{t} A_{k}^{5}\right]+\epsilon^{i j k 0} \partial_{j}\left[\left(b_{\alpha} x^{\alpha}\right) \partial_{k} A_{0}+\left(m_{\alpha} x^{\alpha}\right) \partial_{k} A_{0}^{5}\right]\right\} .
$$

Defining the $i$ th components of vectors $\mathbf{P}$ and $\mathbf{M}$ as

$$
\begin{gathered}
P^{i}=-\frac{n e^{2}}{2 \pi^{2}} \epsilon^{0 i j k}\left[\left(b_{\alpha} x^{\alpha}\right) \partial_{j} A_{k}+\left(m_{\alpha} x^{\alpha}\right) \partial_{j} A_{k}^{5}\right] \\
M^{i}=\frac{n e^{2}}{2 \pi^{2}} \epsilon^{0 i j k}\left[\left(b_{\alpha} x^{\alpha}\right)\left(\partial_{t} A_{k}-\partial_{k} A_{0}\right)+\left(m_{\alpha} x^{\alpha}\right)\left(\partial_{t} A_{k}^{5}-\partial_{k} A_{0}^{5}\right)\right],
\end{gathered}
$$

one thus finds

$$
\mathbf{j}=\partial_{t} \mathbf{P}+\nabla \times \mathbf{M}
$$

Note that the polarization vector $\mathbf{P}$ and the magnetization vector $\mathbf{M}$ can also be written as

$$
\mathbf{P}=\frac{n e^{2}}{2 \pi^{2}}\left[\left(b_{\alpha} x^{\alpha}\right) \mathbf{B}+\left(m_{\alpha} x^{\alpha}\right) \mathbf{B}_{5}\right]
$$

and

$$
\mathbf{M}=\frac{n e^{2}}{2 \pi^{2}}\left[\left(b_{\alpha} x^{\alpha}\right) \mathbf{E}+\left(m_{\alpha} x^{\alpha}\right) \mathbf{E}_{5}\right]
$$

[1] P. Hosur and X. Qi, C. R. Phys. 14, 857 (2013).

[2] H. Weng, X. Dai, and Z. Fang, J. Phys.: Condens. Matter 28, 303001 (2016).

[3] A. A. Burkov, arXiv:1704.06660 [cond-mat.mes-hall].

[4] N. P. Armitage, E. J. Mele, and A. Vishwanath, arXiv:1705.01111 [cond-mat.str-el] .

[5] D. Xiao, M. C. Chang, and Q. Niu, Rev. Mod. Phys. 82, 1959 (2010).

[6] G. E. Volovik, The Universe in a Helium Droplet (Oxford University Press, Oxford, UK, 2003).

[7] A. Vilenkin, Phys. Rev. D 22, 3080 (1980).

[8] K. Fukushima, D. E. Kharzeev, and H. J. Warringa, Phys. Rev. D 78, 074033 (2008).

[9] A. G. Grushin, Phys. Rev. D 86, 045001 (2012).

[10] A. A. Zyuzin and A. A. Burkov, Phys. Rev. B 86, 115133 (2012).

[11] J. Zhou, H. Jiang, Q. Niu, and J. Shi, Chin. Phys. Lett. 30, 027101 (2013).

[12] M. M. Vazifeh and M. Franz, Phys. Rev. Lett. 111, 027201 (2013).

[13] P. Goswami and S. Tewari, Phys. Rev. B 88, 245107 (2013).

[14] K. Landsteiner, Phys. Rev. B 89, 075124 (2014).

[15] M.-C. Chang and M.-F. Yang, Phys. Rev. B 92, 205201 (2015).

[16] J. Ma and D. A. Pesin, Phys. Rev. B 92, 235205 (2015).

[17] S. Zhong, J. E. Moore, and I. Souza, Phys. Rev. Lett. 116, 077201 (2016).
[18] Y. Alavirad and J. D. Sau, Phys. Rev. B 94, 115160 (2016).

[19] P. Baireuther, J. Hutasoit, J. Tworzydło, and C. Beenakker, New J. Phys. 18, 045009 (2016).

[20] J. Ma and D. A. Pesin, Phys. Rev. Lett. 118, 107401 (2017).

[21] R. Shindou and N. Nagaosa, Phys. Rev. Lett. 87, 116801 (2001).

[22] K.-Y. Yang, Y.-M. Lu, and Y. Ran, Phys. Rev. B 84, 075129 (2011).

[23] S. A. Yang, H. Pan, and F. Zhang, Phys. Rev. Lett. 115, 156603 (2015).

[24] Q.-D. Jiang, H. Jiang, H. Liu, Q.-F. Sun, and X. C. Xie, Phys. Rev. Lett. 115, 156602 (2015).

[25] S. A. Parameswaran, T. Grover, D. A. Abanin, D. A. Pesin, and A. Vishwanath, Phys. Rev. X 4, 031035 (2014).

[26] H. Nielsen and M. Ninomiya, Phys. Lett. B 130, 389 (1983).

[27] D. T. Son and B. Z. Spivak, Phys. Rev. B 88, 104412 (2013).

[28] H.-J. Kim, K.-S. Kim, J.-F. Wang, M. Sasaki, N. Satoh, A. Ohnishi, M. Kitaura, M. Yang, and L. Li, Phys. Rev. Lett. 111, 246603 (2013).

[29] A. A. Burkov, Phys. Rev. Lett. 113, 247203 (2014).

[30] J. Xiong, S. K. Kushwaha, T. Liang, J. W. Krizan, M. Hirschberger, W. Wang, R. J. Cava, and N. P. Ong, Science 350, 413 (2015).

[31] X. Huang, L. Zhao, Y. Long, P. Wang, D. Chen, Z. Yang, H. Liang, M. Xue, H. Weng, Z. Fang, X. Dai, and G. Chen, Phys. Rev. X 5, 031023 (2015). 
[32] C.-Z. Li, L.-X. Wang, H. Liu, J. Wang, Z.-M. Liao, and D.-P. Yu, Nat. Commun. 6, 10137 (2015).

[33] H. Li, H. He, H.-Z. Lu, H. Zhang, H. Liu, R. Ma, Z. Fan, S.-Q. Shen, and J. Wang, Nat. Commun. 7, 10301 (2016).

[34] Q. Li, D. E. Kharzeev, C. Zhang, Y. Huang, I. Pletikosic, A. V. Fedorov, R. D. Zhong, J. A. Schneeloch, G. D. Gu, and T. Valla, Nat. Phys. 12, 550 (2016).

[35] C.-L. Zhang, S.-Y. Xu, I. Belopolski, Z. Yuan, Z. Lin, B. Tong, G. Bian, N. Alidoust, C.-C. Lee, S.-M. Huang, T.-R. Chang, G. Chang, C.-H. Hsu, H.-T. Jeng, M. Neupane, D. S. Sanchez, H. Zheng, J. Wang, H. Lin, C. Zhang, H.-Z. Lu, S.-Q. Shen, T. Neupert, M. Zahid Hasan, and S. Jia, Nat. Commun. 7, 10735 (2016).

[36] G. Xu, H. Weng, Z. Wang, X. Dai, and Z. Fang, Phys. Rev. Lett. 107, 186806 (2011).

[37] C. Fang, M. J. Gilbert, X. Dai, and B. A. Bernevig, Phys. Rev. Lett. 108, 266802 (2012).

[38] S.-M. Huang, S.-Y. Xu, I. Belopolski, C.-C. Lee, G. Chang, T.-R. Chang, B. Wang, N. Alidoust, G. Bian, M. Neupane, D. Sanchez, H. Zheng, H.-T. Jeng, A. Bansil, T. Neupert, H. Lin, and M. Z. Hasan, Proc. Natl. Acad. Sci. USA 113, 1180 (2016).

[39] S.-K. Jian and H. Yao, Phys. Rev. B 92, 045121 (2015).

[40] H.-H. Lai, Phys. Rev. B 91, 235131 (2015).

[41] S. Ahn, E. H. Hwang, and H. Min, Sci. Rep. 6, 34023 (2016).

[42] B. Roy, P. Goswami, and V. Juričić, Phys. Rev. B 95, 201102 (2017).

[43] X. Dai, H.-Z. Lu, S.-Q. Shen, and H. Yao, Phys. Rev. B 93, 161110 (2016).

[44] X. Li, B. Roy, and S. Das Sarma, Phys. Rev. B 94, 195144 (2016).

[45] S. Park, S. Woo, E. J. Mele, and H. Min, Phys. Rev. B 95, 161113 (2017).

[46] Q. Chen and G. A. Fiete, Phys. Rev. B 93, 155125 (2016).

[47] R. A. Bertlmann, Anomalies in Quantum Field Theory (Oxford University Press, Oxford, UK, 2000), Vol. 91.

[48] K. Fujikawa and H. Suzuki, Path Integrals and Quantum Anomalies (Oxford University Press on Demand, Oxford, UK, 2004).

[49] D. T. Son and N. Yamamoto, Phys. Rev. D 87, 085016 (2013).

[50] N. Levy, S. Burke, K. Meaker, M. Panlasigui, A. Zettl, F. Guinea, A. C. Neto, and M. Crommie, Science 329, 544 (2010).

[51] R. Jackiw and S.-Y. Pi, Phys. Rev. Lett. 98, 266402 (2007).

[52] M. A. Vozmediano, M. Katsnelson, and F. Guinea, Phys. Rep. 496, 109 (2010).

[53] C.-X. Liu, P. Ye, and X.-L. Qi, Phys. Rev. B 87, 235306 (2013).

[54] A. Cortijo, Y. Ferreirós, K. Landsteiner, and M. A. H. Vozmediano, Phys. Rev. Lett. 115, 177202 (2015).
[55] T. Liu, D. I. Pikulin, and M. Franz, Phys. Rev. B 95, 041201 (2017).

[56] H. Sumiyoshi and S. Fujimoto, Phys. Rev. Lett. 116, 166601 (2016).

[57] A. G. Grushin, J. W. F. Venderbos, A. Vishwanath, and R. Ilan, Phys. Rev. X 6, 041046 (2016).

[58] D. I. Pikulin, A. Chen, and M. Franz, Phys. Rev. X 6, 041021 (2016).

[59] H. Shapourian, T. L. Hughes, and S. Ryu, Phys. Rev. B 92, 165131 (2015).

[60] M. Zubkov, Ann. Phys. 360, 655 (2015).

[61] M. N. Chernodub and M. A. Zubkov, Phys. Rev. B 95, 115410 (2017).

[62] E. V. Gorbar, V. A. Miransky, I. A. Shovkovy, and P. O. Sukhachov, Phys. Rev. Lett. 118, 127601 (2017).

[63] G. Volovik and V. A. Konyshev, JETP Lett. 47, 250 (1988).

[64] D. J. Thouless, M. Kohmoto, M. P. Nightingale, and M. den Nijs, Phys. Rev. Lett. 49, 405 (1982).

[65] J. Zhou, H.-R. Chang, and D. Xiao, Phys. Rev. B 91, 035114 (2015).

[66] A. Cortijo, D. Kharzeev, K. Landsteiner, and M. A. H. Vozmediano, Phys. Rev. B 94, 241405 (2016).

[67] D. T. Son and A. R. Zhitnitsky, Phys. Rev. D 70, 074018 (2004).

[68] M. A. Metlitski and A. R. Zhitnitsky, Phys. Rev. D 72, 045011 (2005).

[69] M. Srednicki, Quantum Field Theory (Cambridge University Press, Oxford, UK, 2007).

[70] W. A. Bardeen, Phys. Rev. 184, 1848 (1969).

[71] J. Fröhlich, R. Götschmann, and P. Marchetti, Commun. Math. Phys. 173, 417 (1995).

[72] X.-L. Qi, T. L. Hughes, and S.-C. Zhang, Phys. Rev. B 78, 195424 (2008).

[73] N. Nagaosa, J. Sinova, S. Onoda, A. H. MacDonald, and N. P. Ong, Rev. Mod. Phys. 82, 1539 (2010).

[74] W. A. Bardeen and B. Zumino, Nucl. Phys. B 244, 421 (1984).

[75] C.-Z. Chang, J. Zhang, M. Liu, Z. Zhang, X. Feng, K. Li, L.-L. Wang, X. Chen, X. Dai, Z. Fang et al., Adv. Mater. 25, 1065 (2013).

[76] D. Xiao, W. Yao, and Q. Niu, Phys. Rev. Lett. 99, 236809 (2007).

[77] A. Rycerz, J. Tworzydlo, and C. W. J. Beenakker, Nat. Phys. 3, 172 (2007).

[78] M. F. Golterman, K. Jansen, and D. B. Kaplan, Phys. Lett. B 301, 219 (1993).

[79] A. J. Niemi and G. W. Semenoff, Phys. Rev. Lett. 51, 2077 (1983). 